\title{
DEMANDAS FÍSICAS SÃO INFLUENCIADAS PELO ESTATUTO POSICIONAL EM PEQUENOS JOGOS DE FUTEBOL?
}

\author{
ARE THE PHYSICAL DEMANDS INFLUENCED BY THE PLAYING POSITION DURING SOCCER \\ SMALL-SIDED GAMES? \\ ¿LAS DEMANDAS FÍSICAS SON INFLUENCIADAS POREL ESTATUTO POSICIONAL EN PEQUEÑOS \\ JUEGOS DEFÚTBOL?
}

Artigo Original

Original ARticle

ARTículo ORIGINAL

Gibson Moreira Praça' ${ }^{1}$
(Profissional de Educação Física)
Igor Júnio de Oliveira Custódio'
(Profissional de Educação Física)
Marcelo Vilhena Silva²
(Profissional de Educação Física)

André Gustavo Pereira de Andrade (Físico)

Pablo Juan Greco'

(Profissional de Educação Física)

1. Universidade Federal de Minas Gerais, Departamento de Esportes, Belo Horizonte, MG.

2. Pontifícia Universidade Católica de Minas Gerais, Belo Horizonte, MG.

\section{Correspondência:}

Pablo Juan Greco

Universidade Federal de Minas

Gerais. Escola de Educação Física,

Fisioterapia e Terapia Ocupacional,

Centro de Estudos em Cognição

e Ação, Universidade Federal de

Minas Gerais. Av. Antônio Carlos,

6627, Belo Horizonte,

MG, Brasil. 31270-901.

pablojgreco@gmail.com

\section{RESUMO}

Introdução: Pequenos jogos são utilizados no treinamento de jogadores de futebol porque replicam as demandas técnicas, táticas, físicas e fisiológicas num contexto semelhante ao do jogo. Contudo, não se sabe se esse modo de treinamento é suficiente para demandar as especificidades observadas no jogo com relação ao estatuto posicional. Objetivo: Comparar a demanda física de defensores, meio-campistas e atacantes durante a realização de pequenos jogos de futebol. Métodos: Dezoito jovens jogadores de futebol (16,4 anos) participaram do estudo, divididos em seis equipes compostas por um defensor, um meio-campista e um atacante. Realizaram-se seis sessões de coleta de dados constituídas por duas séries de quatro minutos com quatro minutos de pausa passiva. Utilizou-se equipamento de GPS de $15 \mathrm{~Hz}$ para coleta das informações. Os dados foram analisados por meio da ANOVA One-way e teste de Kruskal-Wallis, com nível de significância em 5\%. Resultados: Observou-se diferença significativa na demanda física de jogadores de diferentes estatutos posicionais. Meio-campistas percorreram maior percentual da distância em velocidades superiores, enquanto defensores apresentaram distância total reduzida, aumento da distância na velocidade inferior e redução na realização de ações de aceleração. Conclusão: Demandas físicas variam durante a realização de pequenos jogos em função do estatuto posicional.

Descritores: futebol; desempenho atlético; educação física e treinamento.

\section{ABSTRACT}

Introduction: Small-sided games are used during soccer players' training because they replicate the technical, tactical, physical, and physiological demands in a context similar to the game. However, it is not known if this training method is sufficient to demand the specificities observed in the game in relation to the playing position. Objective: To compare the physical demand of defenders, midfielders, and forwards during soccer small-sided games. Methods: Eighteen young soccer players (16,4 years old) participated in this study, divided into six teams composed of a defender, a midfielder, and a forward. Six data collection sessions were performed consisting of two four-minute series with four minutes of passive recovery. A $15 \mathrm{~Hz}$ GPS equipment was used to collect information. Data were analyzed using one-way ANOVA and Kruskal-Wallis test, with a significance level of 5\%. Results: Physical demand was significantly different among players of different playing positions. Midfielders covered a greater percentage of the distance at higher speeds, while defenders covered reduced total distance, increased distance at the lower speed and reduced acceleration actions. Conclusion: During soccer small-sided games, physical demands vary due to the playing position.

Keywords: soccer; athletic performance; physical education and training.

\section{RESUMEN}

Introducción: Pequeños juegos se utilizan en el entrenamiento de jugadores de fútbol porque reproducen las demandas técnicas, tácticas, físicas y fisiológicas en un contexto similar al juego. Sin embargo, se desconoce si ese modo de entrenamiento es suficiente para demandar las especificidades observadas en el juego con relación a la posición ocupada. Objetivo: Comparar la demanda física de defensores, mediocampistas y atacantes durante la realización de pequeños partidos de fútbol. Métodos: Dieciocho jóvenes jugadores de fútbol (16,4 años) participaron del estudio, divididos en seis equipos compuestos por un defensor, un mediocampista y un atacante. Se realizaron seis sesiones de recolección de datos compuestas por dos series de cuatro minutos con cuatro minutos de pausa pasiva. Se utilizó un equipo de GPS de $15 \mathrm{~Hz}$ para la recolección de la información. Los datos fueron analizados por medio de la ANOVA One-way y la prueba Kruskal-Wallis, con un nivel de significación del 5\%. Resultados: Se observó una diferencia significativa en la demanda física de jugadores de diferentes posiciones. Los mediocampistas recorrieron mayor porcentaje de la distancia a velocidades superiores, mientras que los defensores presentaron una distancia total reducida, un aumento de la distancia en la velocidad inferiory la reducción en la realización de acciones de aceleración. Conclusión: Las demandas físicas varían durante la realización de pequeños juegos en función de la posición ocupada en el partido.

Descriptores: fútbol; rendimiento atlético; educación y entrenamiento físico. 


\section{INTRODUÇÃO}

O futebol é um jogo esportivo coletivo caracterizado pela interdependência dinâmica de componentes técnicos, táticos e físicos em um contexto tático-situacional ${ }^{1-3}$. De forma a permitir maior especificidade das sessões de treinamento, recentemente propõem-se pequenos jogos como meios para aprimoramento de capacidades técnicas, físicas e psicológicas ${ }^{4}$ em um contexto imprevisível, aleatório e complexo ${ }^{1}$ caraterístico desta modalidade.

Para o desenvolvimento da qualidade de jogo das equipes, estabelecem-se especificidades na atuação dos jogadores em diferentes posições no campo. Tais especificidades tem sido investigadas sob a alcunha de estatuto posicional5-8. Neste ponto, acredita-se haver demandas específicas - tanto físicas quanto técnico-táticas - para atuação dos jogadores enquanto defensores, meio-campistas ou atacantes, por exemplo.

No contexto de jogo, Di Salvo et al. ${ }^{7}$ (2007), apontaram para uma maior demanda física para meio-campistas em relação a defensores, resultado corroborado por outros estudos ${ }^{9,10}$. Em outro estudo, identificou-se ainda que tais demandas sofreram alterações ao longo dos últimos anos no futebol europeu, nomeadamente entre atacantes ${ }^{11}$. Do ponto de vista tático, estudos observaram diferenças no comportamento de jogadores durante a realização de estruturas na configuração $3 \times 3^{5,6,12}$. Contudo, do ponto de vista físico, desconhece-se até o presente momento se pequenos jogos demandam comportamentos específicos em relação ao estatuto posicional, dado que limita a utilização deste meio no processo de treinamento das capacidades físicas de atletas de futebol.

Em relação aos pequenos jogos, estudos frequentemente investigam respostas técnicas ${ }^{13}$, táticas $^{14}$, físicas - associadas ao perfil motor - e fisiológicas ${ }^{15}$ a partir de alterações nas configurações, i.e. tamanho do campo $^{13}$, número de jogadores ${ }^{13}$, limitação de toques na bola ${ }^{16}$ e superioridade numérica ${ }^{17}$. Em tais estudos, equipes são compostas levando em conta critérios técnicos ${ }^{17}$, nível de capacidade aeróbica ${ }^{17,18}$, ou de maneira aleatória ${ }^{19,20}$, mas poucos adotam a posição de origem na composição das equipes ${ }^{4,18}$, embora o condicionamento físico dos jogadores de futebol deva ser pensado também em função das especificidades demandadas por jogadores de diferentes posições ${ }^{7,9}$.

Conforme apresentado acima, observa-se a importância da utilização dos pequenos jogos no processo de treinamento de jogadores de futebol. Além disso, evidencia-se uma lacuna no que se refere ao conhecimento acerca das demandas físicas em função do estatuto posicional durante pequenos jogos, o que limita a utilização deste meio para o condicionamento de jogadores de futebol. Desta forma, este estudo objetiva comparar a demanda física de jovens jogadores de futebol (defensores, meio-campistas e atacantes) durante a realização de pequenos jogos.

\section{MÉTODOS}

Este estudo recebeu parecer favorável no Comitê de Ética em Pesquisa da Universidade Federal de Minas Gerais, registrado sob o protocolo no 29215814.8.0000.5149. Todos participantes, bem como representantes legais, concederam consentimento livre e esclarecido por escrito

Participaram deste estudo 18 jovens atletas de futebol (idade média $16,4 \pm 0,7$ anos), membros de uma equipe de alto nível de rendimento da cidade de Belo Horizonte com 4,2 anos de média de participação em competições estaduais. O treino padrão consiste de 6-8 sessões semanais com duração de aproximadamente 90 minutos, além dos jogos oficiais.

Este estudo foi conduzido num período de quatro semanas durante os meses de abril e maio de 2014. Todos os formatos de pequenos jogos realizaram-se no formato 3 vs.3 mais goleiros. Os jogadores foram familiarizados com as configurações de pequenos jogos e com os equipamentos de coleta de dados durante a semana número 1. Nas semanas dois a quatro, os jogadores realizaram os pequenos jogos em igualdade numérica (3vs.3), com intervalo de pelo menos 48 h entre eles, em um campo de grama natural nos mesmos horários do dia para minimizar os efeitos do ciclo circadiano ${ }^{21}$.

Reporta-se na literatura diferença nos comportamentos físicos durante partidas de Futebol ${ }^{7}$. Por este motivo, os times foram balanceados em relação à posição de origem dos jogadores (estatuto posicional), sendo cada time composto por um goleiro (não avaliado), um defensor, um meio-campista e um atacante. Um segundo critério adotado na composição das equipes foi o nível de conhecimento tático processual (CTP) dos jogadores. O nível de CTP dos jogadores foi acessado a partir do Teste de Conhecimento Tático Processual: Orientação Esportiva (TCTP:OE) 22, realizado na primeira sessão de coleta de dados. Após isso, dividiram-se os atletas em três grupos de acordo com a posição de origem (defensores, meio-campistas e atacantes) e dentro de cada grupo estabeleceu-se um ranking de acordo com o desempenho no Teste de Conhecimento Tático Processual. Ao final, os 18 jogadores foram alocados em seis equipes (Time 1 a Time 6) (Quadro 1).

Diante da reportada diferença no comportamento dos jogadores em função do confronto equipes de nível inferior ${ }^{23}$, equipes de 1 a 3 não enfrentaram equipes de 4 a 6 e vice-versa.

Cada sessão iniciou-se com 15 minutos de atividade preparatória padrão, composta por corridas, acelerações e contatos com a bola, seguida de duas séries de pequenos jogos. Similarmente ao previamente reportado na literatura, adotou-se a duração de quatro minutos com tempo igual de pausa passiva ${ }^{24}$, permitindo consumo de água ad libitum durante os intervalos. Todas séries de pequenos jogos foram realizadas em um campo com dimensões de $36 \mathrm{~m} \times 27 \mathrm{~m}$ com balizas de $6 \mathrm{~m} \times 2 \mathrm{~m}$, conforme utilizado anteriormente ${ }^{25}$. Durante os pequenos jogos observaram-se todas as regras inerentes ao jogo formal, inclusive o impedimento.

Durante a coleta dos dados, todas equipes enfrentaram-se uma vez (dentro do grupo de nível tático semelhante). Ao todo, observaram-se seis confrontos, 12 séries e 72 observações individuais (24 de defensores, 24 de meio-campistas e 24 de atacantes) em cada uma das variáveis dependentes.

Utilizou-se sistema de posicionamento global (GPS) individual, modelo SPI-Pro X2 (GPSports, SPI-Pro X, Canberra, Austrália), que apresenta um acelerômetro triaxial de $100 \mathrm{~Hz}$ e monitora distâncias e posições a uma frequência de $15 \mathrm{~Hz}$, apresentando suficiente acurácia para ações de aceleração e distância percorrida no futebol ${ }^{26}$. O dispositivo de GPS foi fixado junto ao tórax dos atletas por meio de vestimenta específica. As seguintes variáveis foram consideradas na análise da demanda física: Distância total percorrida; \% da distância total percorrida em velocidades entre 0-7,2 km/h (DT1), entre 7,3-14,3Km/h (DT2), entre 14,4-21,5 Km/h (DT3), e entre $21,5 \mathrm{~km} / \mathrm{h}$ e $25,4 \mathrm{~km} / \mathrm{h}$ (DT4), similarmente ao adotado anteriormente ${ }^{27}$. Já em relação às acelerações, quantificou-se o total de ações de aceleração acima de $2 \mathrm{~m} / \mathrm{s}^{2}$ (AC1Ações), e acima de $2,5 \mathrm{~m} / \mathrm{s}^{2}$ (AC2Ações) e \% da distância total percorrida em acelerações acima de 2, $\mathrm{m} / \mathrm{s}^{2}$ (AC1Dist) e acima de 2,5 m/s $\mathrm{s}^{2}$ (AC2Dist). Analisou-se ainda a aceleração máxima registrada (ACMáx) e a velocidade máxima registrada (VelMáx).

Quadro 1. Composição das Equipes.

\begin{tabular}{|c|c|c|c|}
\hline & Defensor & Meio-campista & Atacante \\
\hline Time 1 & $D^{1}$ & $M^{3}$ & $A^{2}$ \\
\hline Time 2 & $D^{2}$ & $M^{1}$ & $A^{3}$ \\
\hline Time 3 & $D^{3}$ & $M^{2}$ & $A^{5}$ \\
\hline Time 4 & $D^{4}$ & $M^{6}$ & $A^{6}$ \\
\hline Time 5 & $D^{5}$ & $M^{4}$ & $A^{4}$ \\
\hline Time 6 & $D^{6}$ & $M^{5}$ & \\
\hline
\end{tabular}

D: Defensor; M: Meio-campista; A: Atacante

Números sobrescritos indicam a posição final no TCTP: OE relativizada pelo estatuto posicional. 


\section{Análise de dados}

Inicialmente procedeu-se ao teste de normalidade de Shapiro-Wilk, o qual não revelou indícios de desvios significativos à normalidade em nenhuma das variáveis investigadas, exceto D4. Na sequência, utilizou-se a ANOVA one-way para comparação da demanda física entre jogadores de diferentes estatutos posicionais ao longo da coleta. Para a variável com desvio à normalidade, utilizou-se o teste de Kruskal-Wallis. Comparações múltiplas pareadas foram realizadas com o teste de Tukey - variáveis com distribuição normal - e post-hoc de Dunn - variável sem distribuição normal. Calculou-se ainda o tamanho do efeito (TE - $h^{2}$ parcial), classificado em pequeno, médio e grande conforme recomendado na literatura ${ }^{28}$ e o poder observado.

Todas as análises se deram com auxílio ao software IBM SPSS Statistics (versão 20; SPSS, Inc., Chicago, IL, USA). Manteve-se o nível de significância em $5 \%$ ao longo de todas as análises.

\section{RESULTADOS}

A Tabela 1 abaixo apresenta a média (desvio-padrão) das variáveis investigadas neste estudo. Apresentam-se ainda os valores do teste exato de Fisher ( $F$ ), significância ( $p$-valor), tamanho do efeito (TE) e poder observado. A variável distância total foi mensurada em metros e a variável velocidade máxima em quilômetros por hora $(\mathrm{km} / \mathrm{h})$. As variáveis relacionadas ao número de ações de aceleração (AC1Ações e AC2Ações) não apresentam unidade de medida, enquanto as demais variáveis (percentuais de distância em intervalos de velocidade - D1,D2, D3 e D4 - e distâncias em aceleração - AC1Dist e AC2Dist) são apresentadas em termos de percentual da distância total percorrida.

Os resultados apresentados acima sugerem diferenças na demanda física de jogadores de diferentes estatutos posicionais durante pequenos jogos. Observou-se uma redução na demanda física dos defensores no que se refere à distância total em relação aos atacantes ( $p=0,001 ; T E$ : grande) e aos meio-campistas ( $p=0,001$; TE: grande). Estes ainda percorreram menor percentual da distância nas intensidades mais elevadas (D3 em relação aos atacantes - $p=0,29 ;$ TE: pequeno- e D4 em relação tanto aos meio-campistas - $p=0,003$; TE: médio). De maneira geral, meio-campistas apresentaram ainda maior demanda física no que se refere às ações de aceleração, em relação aos defensores, até 2,0m/ $\mathrm{s}^{2}$ ( $p=0,001 ;$ TE: médio) e até $2,5 \mathrm{~m} / \mathrm{s}^{2}$ ( $p=0,050$; TE: pequeno). Observou-se também tal diferença no que se refere à distância percorrida em aceleração no nível 1 em relação aos defensores ( $p=0,001 ; T E ;$ médio) e no nível 2 em relação aos atacantes ( $p=0,050$; TE: pequeno). Por fim, meio-campistas atingiram maior velocidade máxima em relação aos defensores ( $p=0,021$; TE: pequeno).

\section{DISCUSSÃO}

Este estudo objetivou comparar a demanda física de jogadores de futebol de diferentes estatutos posicionais durante a realização de pequenos jogos. De maneira geral, observou-se haver diferenças no perfil motor no que se refere às distâncias, acelerações e velocidades alcançadas por defensores, meio-campistas e atacantes na configuração de jogo 3 vs.3. Verificou-se que os defensores apresentaram reduzida demanda física em relação às demais posições, principalmente em relação aos meio-campistas, os quais apresentaram aumento na demanda física em relação aos defensores e atacantes em diversas variáveis investigadas.

O estatuto posicional tem sido investigado no futebol do ponto de vista das respostas táticas ${ }^{5,6}$ e físicas ${ }^{7,10}$. No que se refere às respostas físicas, Di Salvo et al.7 apontaram que meio-campistas percorrem maior distância total em jogos oficiais do que defensores e atacantes. Similarmente ao atual estudo, acredita-se que a participação efetiva dos jogadores de meio-campo tanto na fase ofensiva quanto defensiva do jogo formal contribui com a elevação da demanda física. Além disso, em outros estudos observou-se redução da distância total percorrida em jogos oficiais por zagueiros em relação aos demais jogadores ${ }^{9,10}$. Tais resultados apontam que além de existir demandas físicas específicas para jogadores de diferentes posições no jogo formal7,9, os pequenos jogos apresentam-se suficientes para reproduzir tais especificidades.

Neste ponto, a novidade do presente resultado incide sob as características dos pequenos jogos. Enquanto estruturas reduzidas em tamanho, mas mantendo a complexidade do jogo formal' ${ }^{12}$, era possível esperar que a ausência de mais jogadores limitasse a especificidade da função em relação aos estatutos posicionais, forçando os atletas a assumir papéis não habituais durante a realização do Pequeno Jogo. Neste sentido, o atual estudo identificou que as estruturas reduzidas aparentemente ainda refletem as especificidades do jogar em função do estatuto posicional, sendo, portanto, potencialmente úteis para o condicionamento físico de jogadores de futebol em função das demandas específicas das posições de origem.

Além disso, reporta-se na literatura influência dos critérios de composição das equipes nas respostas físicas durante pequenos jogos ${ }^{29}$. Dada esta influência, os resultados do presente estudo não podem ser desvinculados da escolha de dividir as equipes mantendo um defensor, um meio-campista e um atacante por time. Assim, acredita-se que tal forma de divisão possa permitir uma manutenção da especificidade da atuação possivelmente não observada em configurações compostas por jogadores de mesmo estatuto posicional, nas quais haveria a necessidade da alteração das demandas usualmente associadas ao estatuto posicional em questão de forma a cumprir com todas nuances táticas inerentes ao jogo.

Tabela 1. Média (desvio-padrão) e valores estatísticos para a comparação do perfil motor entre jogadores de diferentes estatutos posicionais

\begin{tabular}{|c|c|c|c|c|c|c|c|}
\hline & Defensores & Meias & Atacantes & $\mathbf{F}$ & p-valor & TE & Poder observado \\
\hline Distância total & $388,52(39,25)$ & $431,98(44,46)^{1}$ & $460,81(33,36)^{1,2}$ & 20,59 & $0,001^{*}$ & $0,374^{a}$ & 0,999 \\
\hline D1 & $45,72(6,63)^{2,3}$ & $38,35(6,42)$ & $36,17(5,61)$ & 15,45 & $0,001^{*}$ & $0,309^{a}$ & 0,999 \\
\hline D2 & $40,87(5,43)$ & $43,56(6,51)$ & $46,44(6,30)^{1}$ & 5,002 & $0,009^{*}$ & $0,127^{c}$ & 0,797 \\
\hline D3 & $12,83(5,00)$ & $15,57(5,52)$ & $16,50(3,89)^{1}$ & 3,716 & $0,029^{*}$ & $0,097^{c}$ & 0,663 \\
\hline D4 & $0,42(0,80)$ & $2,28(2,14)^{1,3}$ & $0,80(1,14)$ & 10,558 & $0,001^{*}$ & $0,234^{b}$ & 0,986 \\
\hline AC1Ações & $6,87(1,91)$ & $9,25(2,78)^{1}$ & $8,5(1,95)^{1}$ & 6,952 & $0,002^{*}$ & $0,168^{b}$ & 0,915 \\
\hline AC1Dist & $15,72(5,14)$ & $22,10(7,15)^{1}$ & $18,45(4,72)$ & 7,734 & $0,001^{*}$ & $0,176^{b}$ & 0,930 \\
\hline AC2Ações & $3,70(1,45)$ & $4,79(1,91)^{1}$ & $3,83(1,27)$ & 3,417 & $0,038^{*}$ & $0,09 c$ & 0,624 \\
\hline AC2Dist & $9,04(4,06)$ & $11,58(5,12)^{3}$ & $8,67(3,50)$ & 3,294 & $0,043^{*}$ & $0,087^{c}$ & 0,607 \\
\hline VelMÁX & $21,52(2,45)$ & $23,49(2,47)^{1}$ & $21,91(2,57)$ & 4,192 & $0,019^{*}$ & $0,108^{c}$ & 0,719 \\
\hline
\end{tabular}

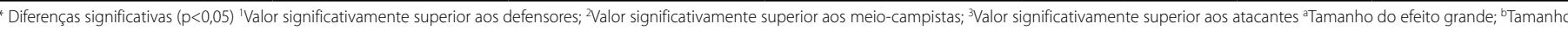
do efeito médio; Tamanho do efeito pequeno. 
Por fim, sugere-se que o treinamento do condicionamento físico de jogadores de futebol esteja em consonância com as demandas previamente especificadas em relação ao estatuto posicional. Desta forma, é possível recomendar pequenos jogos como meio de treinamento por oferecer, além de estímulos específicos ao condicionamento ${ }^{17}$, demandas técnico-táticas ${ }^{2}$ em contexto tático situacional ${ }^{4}$ semelhante ao jogo formal, apresentando-se portanto como importante alternativa aos meios tradicionais para o condicionamento físico de jogadores de futebol já estabelecidos na literatura ${ }^{3}$. Recomenda-se ainda que investigações acadêmicas acerca de configurações de pequenos jogos considerem o estatuto posicional como importante variável para a composição das equipes.

\section{CONCLUSÃO}

Conclui-se haver diferenças na demanda física de jogadores de futebol de diferentes estatutos posicionais durante a prática de pequenos jogos. Especificamente, defensores apresentaram redução na distância percorrida, além de maior percentual da distância percorrida na menor intensidade (até $7,2 \mathrm{~km} / \mathrm{h}$ ). Por outro lado, similarmente ao anteriormente reportado em relação ao jogo formal, meio-campistas apresentaram maior demanda física tanto no que se refere à distância percorrida quanto às acelerações durante a realização de pequenos jogos na configuração 3vs.3.

\section{AGRADECIMENTOS}

Aos profissionais do Clube Atlético Mineiro pelo suporte operacional e científico para coleta dos dados e à Fundação de Amparo à Pesquisa do Estado de Minas Gerais (FAPEMIG), Coordenação de Aperfeiçoamento de Pessoal de Nível Superior (CAPES) e Pró-reitoria de Pesquisa (PRPQ) da Universidade Federal de Minas Gerais pelo suporte financeiro.

Todos os autores declararam não haver qualquer potencial conflito de interesses referente a este artigo.

CONTRIBUIÇÕES DOS AUTORES: Cada autor contribuiu individual e significativamente para o desenvolvimento do manuscrito. GMP (0000-0001-9971-7308)*, PJG $(0000-0003-2607-5935)^{*}$ e AGA (0000-0003-3406-4558)*, foram os principais contribuintes na redação do manuscrito. GMP, IOC (0000-0002-7570-2694)* e MVS (00000001-9978-6768)*, participaram da coleta de dados, importação e análise dos dados dos equipamentos de GPS e revisão crítica do trabalho. AGA e PJG, avaliaram os dados da análise estatística. GP, IC e MS realizaram a pesquisa bibliográfica, a revisão do manuscrito e contribuíram com o conceito intelectual do estudo. *ORCID (Open Researcher and Contributor ID).

\section{REFERÊNCIAS}

1. Garganta JM. Trends of tactical performance analysis in team sports: bridging the gap between research, training and competition. Rev Port Cien Desp. 2009;9(1) 81-9.

2. Aguiar M, Botelho G, Lago C, Maças V, Sampaio J. A Review on the Effects of Soccer Small-Sided Games. J Hum Kinet. 2012;33:103-13.

3. Silva PRS, Romano A, Yazbek P, Battistella LR. Efeito do treinamento físico específico nas respostas cardiorrespiratórias e metabólicas em repouso e no exercício máximo em jogadores de futebol profissional. Rev Bras Med Esporte; 1997;3(4):101-7.

4. Praça GM, Custódio IJO, Greco PJ. Numerical superiority changes the physical demands of soccer players during small-sided games. Rev Bras Cineantropom Desemp Hum. 2015;17(3):269-79.

5. Padilha MB, Moraes JC, Costa IT. O estatuto posicional pode influenciar o desempenho tático entre jogadores da Categoria Sub-13? Rev Bras Ci Mov. 2013;21(4):73-9.

6. Silva BSR. Estudo dos comportamentos táticos de jogadores de Futebol em jogos reduzidos e por estatuto posicional [dissertação]. Porto, Lisboa: Universidade do Porto, Faculdade de Desporto; 2011.

7. Di Salvo V, Baron R, Tschan H, Calderon Montero FJ, Bachl N, Pigozzi F. Performance characteristics according to playing position in elite soccer. Int J Sports Med. 2007;28(3):222-7.

8. Teplan J, Malý T, Zahálka F, Malá L. The level and relationship between speed and agility according to the playing roles in elite young soccer players. Gazz Med Ital . 2014;173(1-2):29-38.

9. Wehbe GM, Hartwig TB, Duncan CS. Movement analysis of Australian national league soccer players using global positioning system technology. J Strength Cond Res. 2014;28(3):834-42.

10. Dellal A, Wong DP, Moalla W, Chamari K. Physical and technical activity of soccer players in the French First League - with special reference to their playing position. Int SportMed J. 2010;11(2):278-90.

11. Bush M, Barnes C, Archer DT, Hogg B, Bradley PS. Evolution of match performance parameters for various playing positions in the English Premier League. Hum Mov Sci. 2015;39(1):1-11.

12. Praça GM. Pequenos Jogos no Futebol: comportamento tático e perfil motor em superioridade numérica [dissertação]. Belo Horizonte, MG: Universidade Federal de Minas Gerais, Escola de Educação Física, Fisioterapia e Terapia Ocupacional; 2014

13. Abrantes $\mathrm{Cl}$, Nunes MI, Maçãs VM, Leite NM, Sampaio JE. Effects of the number of players and game type constraints on heart rate, rating of perceived exertion, and technical actions of small-sided soccer games. J Strength Cond Res. 2012;26(4):976-81.

14. Folgado H, Lemmink KA, Frencken W, Sampaio J. Length, width and centroid distance as measures of teams tactical performance in youth football. Eur J Sport Sci. 2014;14(Suppl 1):S487-92.

15. Aguiar MV, Botelho GM, Gonçalves BS, Sampaio JE. Physiological responses and activity profiles of football small-sided games. J Strength Cond Res. 2013;27(5):1287-94.
16. Casamichana D, Román-Quintana JS, Gonzalez JC, Castellano J. Use of limiting the number of touches of the ball in soccer training: Does it affect the physical and physiological demands?. Rev Int Cienc Deporte; 2013;9(33):208-21

17. Hill-Haas SV, Dawson BT, Coutts AJ, Rowsell GJ. Physiological responses and time-motion characteristics of various small-sided soccer games in youth players. J Sports Sci. 2009;27(1):1-8.

18. Casamichana D, Castellano J, Dellal A. Influence of different training regimes on physical and physiologica demands during small-sided soccer games: continuous vs. intermittent format. J Strength Cond Res. 2013;27(3):690-7.

19. Jones S, Drust B. Physiological and technical demands of $4 \vee 4$ and $8 \vee 8$ games in elite youth soccer players. Kinesiology. 2007;39(2):150-6

20. Katis A, Kellis E. Effects of small-sided games on physical conditioning and performance in young soccer players. J Sports Sci Med. 2009;8(3):374-80

21. Drust B, Waterhouse J, Atkinson G, Edwards B, Reilly T. Circadian rhythms in sports performance--an update. Chronobiol Int. 2005;22(1):21-44.

22. Greco PJ, Aburachid LMC, Silva SR, Morales JCP. Validação de conteúdo de ações tático-técnicas do Teste de Conhecimento Tático Processual - Orientação Esportiva. Motricidade. 2014;10(1):38-48.

23. Folgado H, Duarte R, Fernandes O, Sampaio J. Competing with lower level opponents decreases intra-team movement synchronization and time-motion demands during pre-season soccer matches. PLoS One. 2014;9(5):e97145.

24. Dellal A, Owen A, Wong DP, Krustrup P, van Exsel M, Mallo J. Technical and physical demand of small vs. large sided games in relation to playing position in elite soccer. Hum Mov Sci. 2012:31(4):957-69.

25. Costa IT, Garganta J, Greco PJ, Mesquita I, Maia J. System of tactical assessment in Soccer (FUT-SAT) Development and preliminary validation. Motricidade. 2011;7(1):69-83.

26. Buchheit M, Al Haddad H, Simpson BM, Palazzi D, Bourdon PC, Di Salvo V, et al. Monitoring accelerations with GPS in football: time to slow down? Int J Sports Physiol Perform. 2014;9(3):442-5.

27. Owen AL, Wong DP, Paul D, Dellal A. Physical and technical comparisons between various-sided games within professional soccer. Int J Sports Med. 2014;35(4):286-92.

28. Pierce CA, Block RA, Aguinis H. Cautionary note on reporting eta-squared values from multifactor anova designs. Educ Psychol Measur. 2004;64(6):916-24.

29. KöklüY, Ersöz G, Alemdaroğlu U, Aşç A, Ozkan A. Physiological responses and time-motion characteristics of 4-a-side small-sided game in young soccer players: the influence of different team formation methods. J Strength Cond Res. 2012;26(11):3118-23. 